\title{
Giant Extra-Ordinary Near Infrared Transmission from Seemingly Opaque Plasmonic Metasurface: Sensing Applications
}

\section{Sagar Kumar Verma}

IIT ROORKEE

Sachin Kumar Srivastava ( $\nabla$ sachin.srivastava@ph.iitr.ac.in )

IIT Roorkee: Indian Institute of Technology Roorkee https://orcid.org/0000-0003-1711-1551

\section{Research Article}

Keywords: Extra-ordinary optical transmission, metasurfaces, surface plasmon, nano-slits, coupled plasmonic mode, sensor

Posted Date: July 15th, 2021

DOI: https://doi.org/10.21203/rs.3.rs-684812/v1

License: (c) (i) This work is licensed under a Creative Commons Attribution 4.0 International License.

Read Full License

Version of Record: A version of this preprint was published at Plasmonics on October 19th, 2021. See the published version at https://doi.org/10.1007/s11468-021-01551-1. 


\title{
Giant Extra-Ordinary Near Infrared \\ Transmission from Seemingly Opaque \\ Plasmonic Metasurface: Sensing applications
}

\author{
Sagar Kumar Verma, Sachin K. Srivastava* \\ Department of Physics, Indian Institute of Technology Roorkee, Roorkee, \\ Haridwar, Uttarakhand-247667, India \\ *Corresponding author \\ Tel.: +91-1332-28-5715 \\ Fax: +91-1332-28-6662 \\ Email: sachin.srivastava@ph.iitr.ac.in
}

\begin{abstract}
:
In the present study, we report giant extra-ordinary transmission of near infra-red (NIR) light, more than $90 \%$, through a seemingly opaque plasmonic metasurface, which consists of two metal nanoslits arrays (MNSAs) with alternate opening arrangements. By using perfect coupling of the plasmonic modes formed between the sharp edges of the upper and lower MNSAs of silver, a giant, wavelength selective transmission could be obtained. The study is accompanied by optimization of electromagnetic (EM) field coupling for different interlayer spacings and lateral overlap between the two MNSAs to understand their significance in light transmission through the metasurface. The interlayer spacing between the MNSAs works as the transmitting channel for light. The optimization of performance with different fill factors and plasmonic metals was performed as well. Because of the excitation of extended surface plasmons (ESPs) generated at both the MNSAs, the metasurface can be used for refractive index (RI) sensing as one of its applications by using a transparent and flexible polymer, such as polydimethylsiloxane (PDMS), as substrate. The maximum sensitivity which could be achieved for the optimal configuration of the metasurface was $1435.71 \mathrm{~nm} / \mathrm{RIU}$, with a figure of merit (FOM) of $80 \mathrm{RIU}^{-1}$ for $90.45 \%$ optical transmission of light for the refractive index variation of analyte medium from 1.33 to 1.38 RIU. The present study strengthens the concept of light funneling through subwavelength structures due to plasmons, which are responsible for light transmission through this seemingly opaque metasurface and finds use in highly sensitive, flexible, and cost effective EOT based sensors.
\end{abstract}

Keywords: Extra-ordinary optical transmission, metasurfaces, surface plasmon, nano-slits, coupled plasmonic mode, sensor 


\section{Introduction}

Plasmonic metasurfaces exhibit light controlling property in the same way as the metamaterials exhibit for the incoming radiation on the structure itself. That's why the metasurfaces are called 2D version of the metamaterials [1-3]. Such metasurfaces are designed with an array of unit metal/dielectric nano-structures having specific geometry, size and orientation [4-6]. Plasmonic metasurfaces offer an ability to control the optical surface waves, such as surface plasmon polaritons (SPPs) in the near filed regime [7-11]. It is possible to manipulate the light propagation and SPPs by using transformation optics approach [12-15]. It has also been explained by several groups that SPPs can be controlled efficiently by specific patterning of the metasurfaces [9-11]. The plasmonic metasurfaces having patterned nanostructures generate SPPs as the part of re-radiative energy with the direct interaction of light. Such plasmonic nanostructures perform as SPPs emitters. By controlling the relative phase among the emitters via modifying the geometry of the nanostructures or the polarization state of the incident light, one can achieve unidirectional excitation of SPPs [7, 12]. Because of amazing light controlling property, the plasmonic metasurfaces having metallic nanostructures of subwavelength size have been used for many applications in photonics, including bio-sensing [16, 17], spectroscopy [18, 19], nanolasing [20], all-optical switching [21], nonlinear optical processes [22], and metasurface technologies [23-25]. Most of the plasmonic metasurfaces possess subwavelength openings as compared to the wavelengths of incident EM wave, where the transmission is based on the principle of Extra-ordinary transmission (EOT) due to excitation of surface plasmons. EOT is the phenomenon in which the intensity of the light transmitted through a plasmonic structure containing subwavelength periodic apertures is much higher than the intensity of light falling on the structure's openings. In 1998, the term EOT 
was coined and experimentally demonstrated by Ebbesen et al.[26]. EOT has extensively been investigated for the last two decades because of its considerable scientific interest and broad potential applications in various technical fields, for example, photolithography[27], near field microscopy [28, 29], light-emitting diodes and lasers [30-33], sensors[34, 35] surface-enhanced Raman spectroscopy (SERS) [36, 37], surface-enhanced IR absorption (SEIRA) [38, 39], surfaceenhanced fluorescence (SEF) [40-43], as a wave plate to control light polarization $[44,45]$, etc.. The EOT is attributed to the excitation of surface plasmons (SPs), which are responsible for funneling the light through the subwavelength aperture[46, 47]. In the history of light transmission through a subwavelength aperture, the transmission efficiencies reported have been very low for a long time; the reason being the evaluation on the basis of very week coupling of radiative electromagnetic modes excited by the subwavelength spacing and decaying evanescent electromagnetic fields inside the subwavelength apertures [48-51]. Hence, strongly coupled plasmonic modes may lead to improved transmission efficiencies. It is possible to achieve optical transmission through subwavelength transmission gratings or metal nano-slits array (MNSA) at wavelengths larger than the period of the structure by optimizing the geometrical parameters such as groove width and height of the MNSA [26, 52]. In another approach, EOT is explained via quasi-cylindrical waves, which are generated at the emerging side of the structure; such waves are generated because of the funneling of propagating surface plasmons [53-55].

Periodic plasmonic nanostructure-based optical sensors are highly promising because of their excellent sensing characteristics such as high sensitivity, large durability, good repeatability, and long shelf life [56, 57]. EOT-based sensing technique can be used in wavelength modulation-based refractive index (RI) 
sensing. It was reported both experimentally and theoretically that plasmonic MNSA could support dual surface plasmon modes, one of which is confined to the substrate does not interact with the analyte medium. In contrast, the second one interacts with the analyte medium, which helps in sensing the change in the analyte medium [58-60]. A self-referenced sensor based on EOT through gold (Au) nanoslits array was designed and both theoretically and experimentally studied by Srivastava and Abdulhalim [61]. They achieved around 30\% transmittance through MNSA. The response of periodic subwavelength plasmonic structures for incoming radiation in reflection or transmission mode depends on geometrical parameters of the structure such as period, size, shape, and surface roughness [62-65]. It was both experimentally and theoretically demonstrated that by tuning the geometrical parameter of periodic grating structure, it is possible to excite various types of cavity modes or even higher order of plasmonic modes with varying sensor sensitivity [66].

In this work, we have focused on the enhancement of light transmission through a seemingly opaque plasmonic metsurface consisting of two MNSAs, and studied the roles of the vertical interlayer distance and later overlap between the upper and lower MNSAs in the enhancement of light transmission. Such enhancement in EOT is possible by the perfect coupling of the plasmonic modes generated by the upper and lower MNSA. The periodic geometrical structure provides the additional phase to match that of surface plasmons supported by the structure to excite plasmonic modes. Such structure, as simulated in this study, can be used for refractive index or polydimethylsiloxanes (PDMS). 


\section{Simulations}

A schematic of the proposed metasurface, consisting of two plasmonic MNSAs has been shown in the Fig. 1 , in which $\Lambda$ is the period, $\mathrm{W}$ is the width of the metal nanoslit, a is groove width of the structure, $\mathrm{H}$ is the vertical distance between upper and lower MNSA, and $\mathrm{h}$ is the thickness of the metal nano-slits. The definitions of $\mathrm{W}$ and a are opposite to each other for the lower MNSA. In all simulations, the period of the structure was kept constant, which is $1600 \mathrm{~nm}$, keeping in mind the experimental demonstration of the same after the opening of the lockdown due to COVID-19 using a compact disk (CD) as a template for its fabrication and hence the height of the metal films has been kept the same for both the MNSAs. The fill factor of the structure is defined by $f=W / \Lambda$, which is also optimized for optimal transmission. EOT through the proposed metasurface was simulated using COMSOL Multiphysics (Version 5.5) under the normal incidence of TM polarized light for the near infra-red (NIR) range of the electromagnetic spectrum from the top side of the structure, and $0^{\text {th }}$ order of diffraction of the light transmitted through the bottom of the plasmonic metasurface was analyzed.

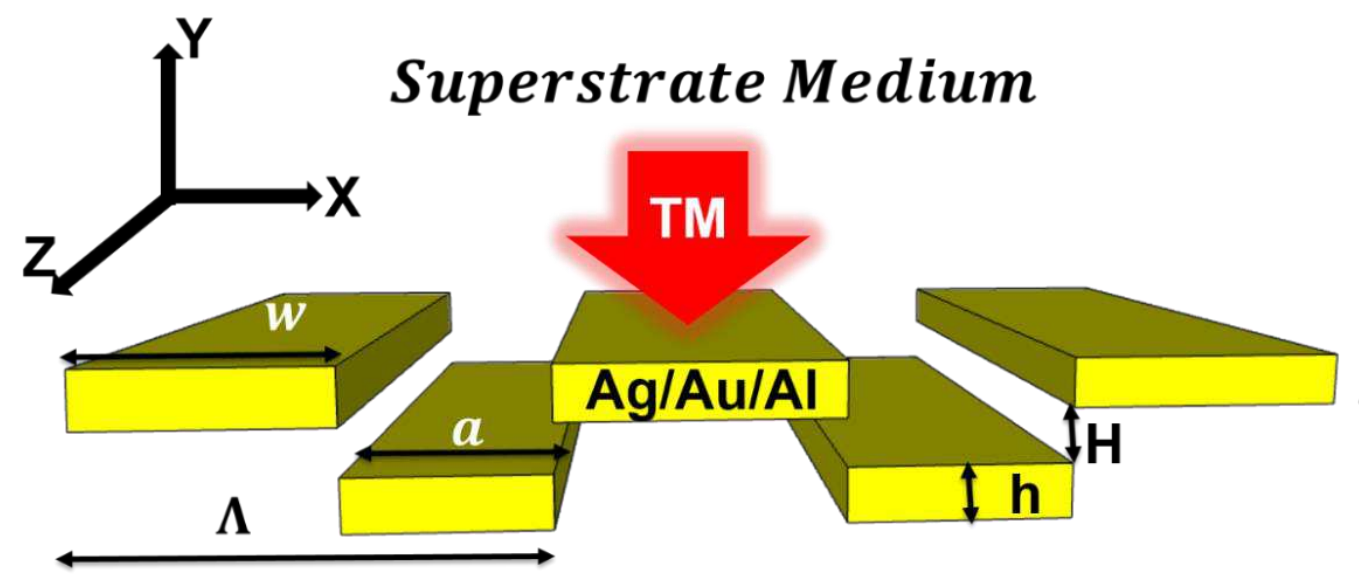

Substrate Medium

Fig. 1 Schematic of the proposed metasurface consisting of two MNSAs separated by 'H' distance 
Extended surface plasmons (ESPs) are supported in both the MNSAs of such a metasurface. The excitation of ESPs in both the MNSAs is governed by the following plasmonic phase matching equation at the metal-dielectric interface [67, $68]$

$$
\operatorname{Re}\left(K_{\text {inc }}\right)+K_{G}= \pm \operatorname{Re}\left(K_{S P}\right)
$$

where, $\operatorname{Re}\left(K_{\text {inc }}\right)$ and $\operatorname{Re}\left(K_{S P}\right)$ are the real parts of the propagation vector of the incident light in the polarization direction and that of surface plasmons, respectively and $K_{G}$ is the k-vector of the periodic structure, which provides the additional phase to achieve SPR condition, and it can be defined by the following equation as

$$
\mathrm{K}_{\mathrm{G}}=\mathrm{nG}_{\mathrm{x}}+\mathrm{mG}_{\mathrm{y}}
$$

where, $G_{x}=G_{y}=2 \pi / \Lambda$ are the grating momentum vectors for a two-dimensional (2D) array of periodic structure, and $\mathrm{n}$ and $\mathrm{m}$ are integers. The propagation vector of SPs, $\operatorname{Re}\left(K_{S P}\right)$ is given[66] as:

$$
\mathrm{K}_{\mathrm{SP}}=\mathrm{K}_{0} \sqrt{\frac{\varepsilon_{\mathrm{m}} \varepsilon_{\mathrm{d}}}{\varepsilon_{\mathrm{m}}+\varepsilon_{\mathrm{d}}}}
$$

where, $\varepsilon_{m}$ and $\varepsilon_{d}$ are the dielectric constants for the metal and the dielectric media, respectively and $K_{0}=2 \pi / \lambda$ is the wave vector of light in free space for incident light of wavelength $\lambda$.After algebraic simplifications using equations (2) and (3), the equation (1) can be re-written in the following form for 1D periodic structure:

$$
\mathrm{K}_{0} \mathrm{n}_{\mathrm{d}} \sin \theta+\mathrm{m}\left(\frac{2 \pi}{\Lambda}\right)= \pm \mathrm{K}_{0} \sqrt{\frac{\operatorname{Re}\left(\varepsilon_{\mathrm{m}}\right) \varepsilon_{\mathrm{d}}}{\operatorname{Re}\left(\varepsilon_{\mathrm{m}}\right)+\varepsilon_{\mathrm{d}}}}
$$

where, $n_{d}\left(=\sqrt{\varepsilon_{d}}\right)$ is the RI of analyte medium, and $\theta$ is the angle of incidence of light. For the normal incidence $\left(\theta=0^{0}\right)$ of light, the ESP resonance wavelength is given by the following equation [66]:

$$
\lambda=\frac{\Lambda}{\mathrm{m}} \sqrt{\frac{\operatorname{Re}\left(\varepsilon_{\mathrm{m}}\right) \varepsilon_{\mathrm{d}}}{\operatorname{Re}\left(\varepsilon_{\mathrm{m}}\right)+\varepsilon_{\mathrm{d}}}}
$$


The value of the dielectric constant $\varepsilon_{d}$ is chosen according to the application, and the first, second or even higher-order resonance wavelengths can be calculated by using equation (5). For $n_{d}=1$, the real part of the dielectric constant of the metal $\operatorname{Re}\left(\varepsilon_{m}\right) \gg \varepsilon_{d}$, the first order resonant wavelength $\lambda$ can analytically be estimated, using equation (5), as $\lambda \sim \Lambda$ and for $\Lambda=1600 \mathrm{~nm}$ the first order resonance wavelength should be around 1600nm individually for both the MNSAs.

The whole simulation work was done by using licensed version of COMSOLMultiphysics version 5.5 based on the principle of finite element method (FEM) of computation. For noble metals $(\mathrm{Ag}, \mathrm{Au}, \mathrm{Al})$, the refractive indices were taken from Johnson and Christy [69], which is also inbuilt in the material database of COMSOL. Electromagnetic wave frequency domain (ewfd.) module for normal incidence of a transverse magnetically polarized (TM) plane electromagnetic wave. along with Floquet periodic boundary conditions [70] for the lateral boundaries was used and perfectly matching layers (PMLs) were applied at the longitudinal boundaries in the simulation window. First, the refractive indices of the superstrate and the substrate was considered air $(n=1)$ to study the coupled-mode behavior of the plasmonic modes excited at the upper and lower MNSAs, responsible for the giant EOT. Using COMSOL, the resonance wavelength for the complete metasurface comprised of coupled MNSAs was obtained to be equal to $1641.2 \mathrm{~nm}$. Control simulations towards the optimization of $\mathrm{h}$ and $\mathrm{H}$ were performed for the maximum possible strength of the transmitted peak for the $0^{\text {th }}$ order of the diffracted light through the metasurface.

To analyze the sensing capabilities of the proposed metasurface, the refractive index of the superstrate medium was varied as that of an analyte medium having RI varying from 1.33 to 1.38 RIU in the steps of 0.01 and the transmission spectra were simulated. After a satisfactory sensing response was obtained for the 
aforementioned metasurface, the free floating substrate medium was replaced with polydimethylsiloxane (PDMS) polymer to make a practically feasible sensor and also because of various useful optical and mechanical properties of PDMS [71, 72] with the same range of refractive index of the analyte medium. The Refractive index $\left(n_{P D M S}\right)$ of PDMS is given by the following equation [73]

$$
\mathrm{n}(\lambda)_{\mathrm{PDMS}}=\sqrt{1+\frac{1.0093 \lambda(\mu \mathrm{m})^{2}}{\lambda(\mu \mathrm{m})^{2}-0.013185}}
$$

where, $\lambda$ is wavelength of the incident light.

Any sensor is characterized by two essential parameters: one, the sensitivity, and the other is the figure of merit (FOM). In the whole study, the wavelength interrogation has been performed to study the spectral response of the proposed structure. The sensitivity of the sensor will be defined as follows [74]:

$$
\mathrm{S}=\frac{\Delta \lambda_{\text {res }}}{\Delta \mathrm{n}_{\mathrm{a}}}
$$

where, $\Delta \lambda_{\text {res }}$ is the shift in plasmonic resonance wavelength with the corresponding change in the refractive index of the analyte medium $\left(\triangle n_{a}\right)$. FOM of the sensor is defined as the ratio of the sensitivity of the sensor to the full width half maximum (FWHM) of the spectral response and can be estimated by the following equation [70]:

$$
\mathrm{FOM}=\frac{\mathrm{S}}{\mathrm{FWHM}}
$$

\section{Results and Discussion}

First, we optimized the proposed structure of metasurface for maximum optical transmission, around $90 \%$ with a fill factor of 0.625 , by changing the thickness $(\mathrm{h})$ of $\mathrm{Ag}$ nano-slits from 10 to $125 \mathrm{~nm}$ and the vertical distance between upper and lower nano-slits array $(\mathrm{H})$ from 125 to $0 \mathrm{~nm}$ (consequently) with refractive index, $\mathrm{n}=1$ for both substrate and superstrate media. 

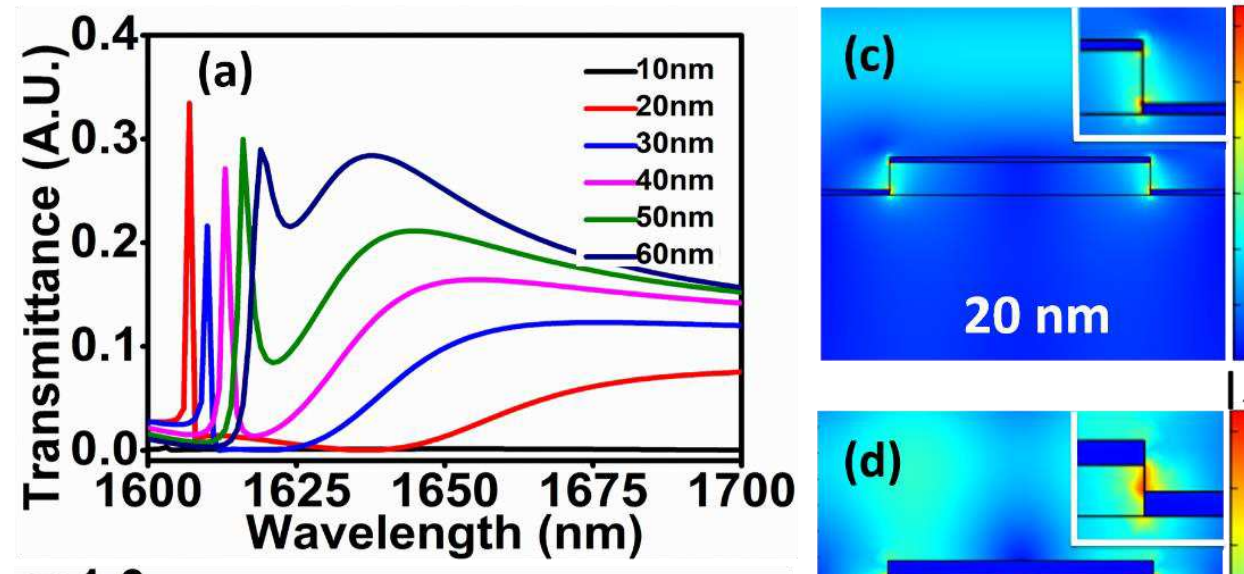

$\left|E / E_{0}\right|$
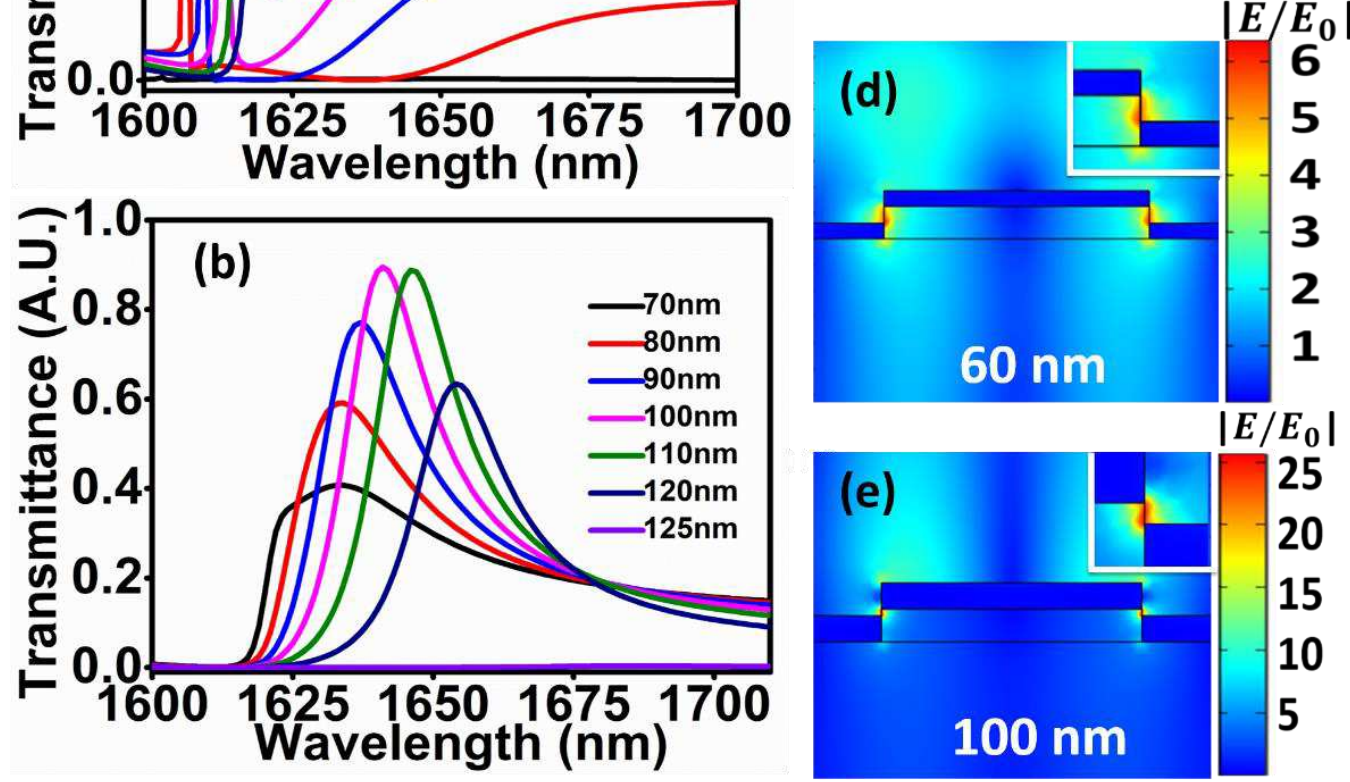

Fig. 2 a \& b Transmission spectra for various thicknesses of Ag metal nano-slits, c- e Electric field profiles for $20 \mathrm{~nm}, 60 \mathrm{~nm}$, and $100 \mathrm{~nm} \mathrm{Ag}$ film thicknesses at respective resonance wavelengths, respectively. The insets in Figures $\mathbf{c}-\mathbf{e}$ show the zoomed in view of the field profile near edges.

In Fig. $2 \mathrm{a} \& \mathrm{~b}$, the transmission spectra for various thickness of Ag have been plotted. Figure 2 a explains that for $20 \mathrm{~nm}$ thickness of the metal film (h), the Transmittance versus Wavelength plot shows two resonance peaks for the $0^{\text {th }}$ order of the transmitted light through the proposed plasmonic metasurface. The two resonance peaks correspond to the two resonant plasmonic modes at $\lambda_{1}(1607 \mathrm{~nm})$ and $\lambda_{2}(1679 \mathrm{~nm})$ each corresponding to the upper and lower MNSAs, respectively. A clearer understanding of the claim that one of the resonances corresponds to each of the MNSAs will follow from the subsequent discussions on Fig.3. For the case of $20 \mathrm{~nm} \mathrm{Ag}$ film thickness, the gap between both the MNSAs, H, was $95 \mathrm{~nm}$, while it was $115 \mathrm{~nm}$ initially when $\mathrm{h}$ was $10 \mathrm{~nm}$. The value of $\mathrm{H}$ decreases with an increase 
in the value of $h$, as the total thickness cannot exceed pertaining to the practically feasible, cost-effective fabrication of the metasurface. With an increase in the value of $\mathrm{h}$ up to $60 \mathrm{~nm}$, both the peaks are observed to shift towards each other. This is a consequence of decrease in the value of $\mathrm{H}$ with an increase in $\mathrm{h}$, which leads to coupling of the plasmonic modes at the sharp edges of the nano-slits of upper and lower MNSAs, as it is evident from the electric field profiles plotted in Fig. 2 c \& d. The insets of these figures give a clear understanding of the electromagnetic (EM) field coupling, while the color bars show the strengths of the electric field. In continuation to the aforementioned discussions of Fig.2 a, c and d, when the thickness of the $\mathrm{Ag}$ film is further increased, leading to a decrease in $\mathrm{H}$, the coupling between the EM modes becomes even more stronger, which leads to merging of both the resonance peaks to one. It can be observed in Fig. $2 \mathrm{~b}$ that when the value of $\mathrm{h}$ was increased from $70 \mathrm{~nm}$ to $125 \mathrm{~nm}$, the merged resonance peak shows a monotonous red shift, while the transmittance first increases to maximum of $90.45 \%$ at $100 \mathrm{~nm}$ film thickness with an increase in the film thickness from $70 \mathrm{~nm}$ to $100 \mathrm{~nm}$ and then starts decreasing with further increase in the film thickness up to $120 \mathrm{~nm}$. At $\mathrm{h}=125 \mathrm{~nm}$, both the MNSAs touch each other by their edges, leading to complete blockage of light and hence, the transmittance becomes zero. The coupling between the plasmonic modes plays an essential role in facilitating a very high value of EOT through the metasurface, which is $90.45 \%$. Figure 2 e shows the electric field profile for metasurface with $100 \mathrm{~nm}$ Ag film thickness at resonance wavelength of $1641.2 \mathrm{~nm}$. The value of electric field normalized by the incident field was much larger for $100 \mathrm{~nm}$ optimum thickness of Ag nano-slits, because of the excitation of perfectly coupled modes at the sharp edges of nano-slits.

After achieving the maximum transmittance of $90.45 \%$ for $100 \mathrm{~nm}$ thickness $(\mathrm{h})$ of Ag nano-slits and interlayer vertical distance $(\mathrm{H}=25 \mathrm{~nm})$, we studied the role of 
variation of $\mathrm{H}$ on the characteristics of the metasurface. The transmission spectra were simulated and have been plotted in Fig. 3 a \& b for decreasing the value of $\mathrm{H}$ from $25 \mathrm{~nm}$ to $0 \mathrm{~nm}$ and increasing it from $25 \mathrm{~nm}$ to $190 \mathrm{~nm}$, respectively, while keeping the Ag-nano slits thickness (h) as 100nm in both the MNSAs.

In Fig. 3 a, it is observed that for each configuration, the transmission vs wavelength plot shows a maximum at a particular wavelength for the reasons explained in the discussions of Fig. 2. It is further observed that with a decrease in the value of $\mathrm{H}$, the transmittance decreases monotonously with a slight red shit in the resonance wavelength. This is attributed to decrease in the opening of the structure for light transmission in the current spectral window of simulation. For $\mathrm{H}=0$, the upper and lower MNSAs touch each other, thereby leading to zero transmission. Figure $3 \mathrm{~b}$ shows the transmission spectra with an increase in the value of $\mathrm{H}$ varying between $25-190 \mathrm{~nm}$ for $\mathrm{h}=100 \mathrm{~nm}$. It can be observed that with an increase in the value of $\mathrm{H}$, the coupled plasmonic resonances start to decouple and hence a slight redshift, accompanied by a decrease in the transmittance is seen with an increase $\mathrm{H}$ from 25 $\mathrm{nm}$ to $75 \mathrm{~nm}$.

To make a better understanding of the role of $\mathrm{H}$ over the metasurface transmittance, we have plotted the EM field profiles for $\mathrm{H}=25,55$ and $75 \mathrm{~nm}$ in figures $3 \mathrm{c}, \mathrm{d} \&$ e, respectively. The insets of the aforementioned figures show the zoomed in views of the EM field profiles near the edges of both the MNSAs. It can be clearly observed that with an increase in the value of $\mathrm{H}$ up to $75 \mathrm{~nm}$, the EM field coupling and hence the value of the normalized field in the hotspots reduces by $1 / 4^{\text {th }}$ of its value for the optimum configuration, which is a consequence of the mode decoupling, thereby leading to decrease in the transmission efficiency. Further, when $\mathrm{H}$ is increased more than $75 \mathrm{~nm}$ up to $190 \mathrm{~nm}$, the coupled modes formed at the sharp edges of upper and lower MNSA start to decouple and show two 

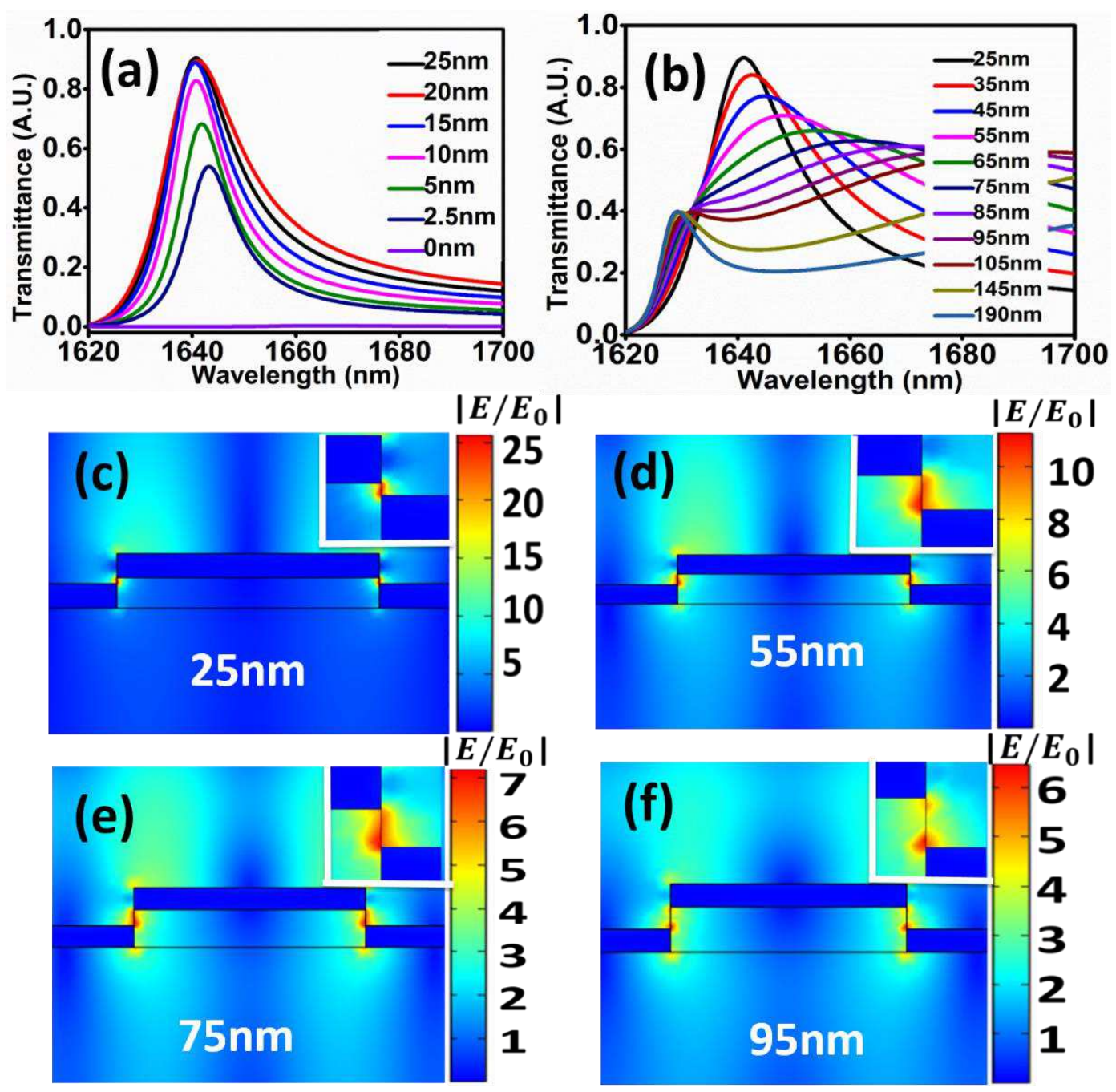

Fig. 3 Transmission spectra for a decreasing -, b increasing - interlayer vertical distance $(\mathrm{H})$ between upper and lower MNSAs; c-f are the electric field profile at shifting resonance wavelength for $\mathrm{H}=$ $25 \mathrm{~nm}, 55 \mathrm{~nm}, 75 \mathrm{~nm}$, and $95 \mathrm{~nm}$, respectively. The insets of $\mathbf{c}-\mathbf{f}$ show the zoomed in view of the EM field enhancement at the edges of both the MNSAs

plasmonic resonances, while the overall transmittance for both the resonances going down. A clear insight of the aforementioned sentence can be seen in Fig. $3 \mathrm{f}$, where the EM fields of both the MNSAs decouple almost completely for $\mathrm{H}=95 \mathrm{~nm}$ and so on. If the value of $\mathrm{H}$ is increased further, the transmittance goes down to zero, ultimately. Hence, it can be concluded that the maximum transmission occurs only when the plasmonic modes generated by upper and lower metal slits array coupled ideally at an optimal combination of $\mathrm{h}$ and $\mathrm{H}$. 


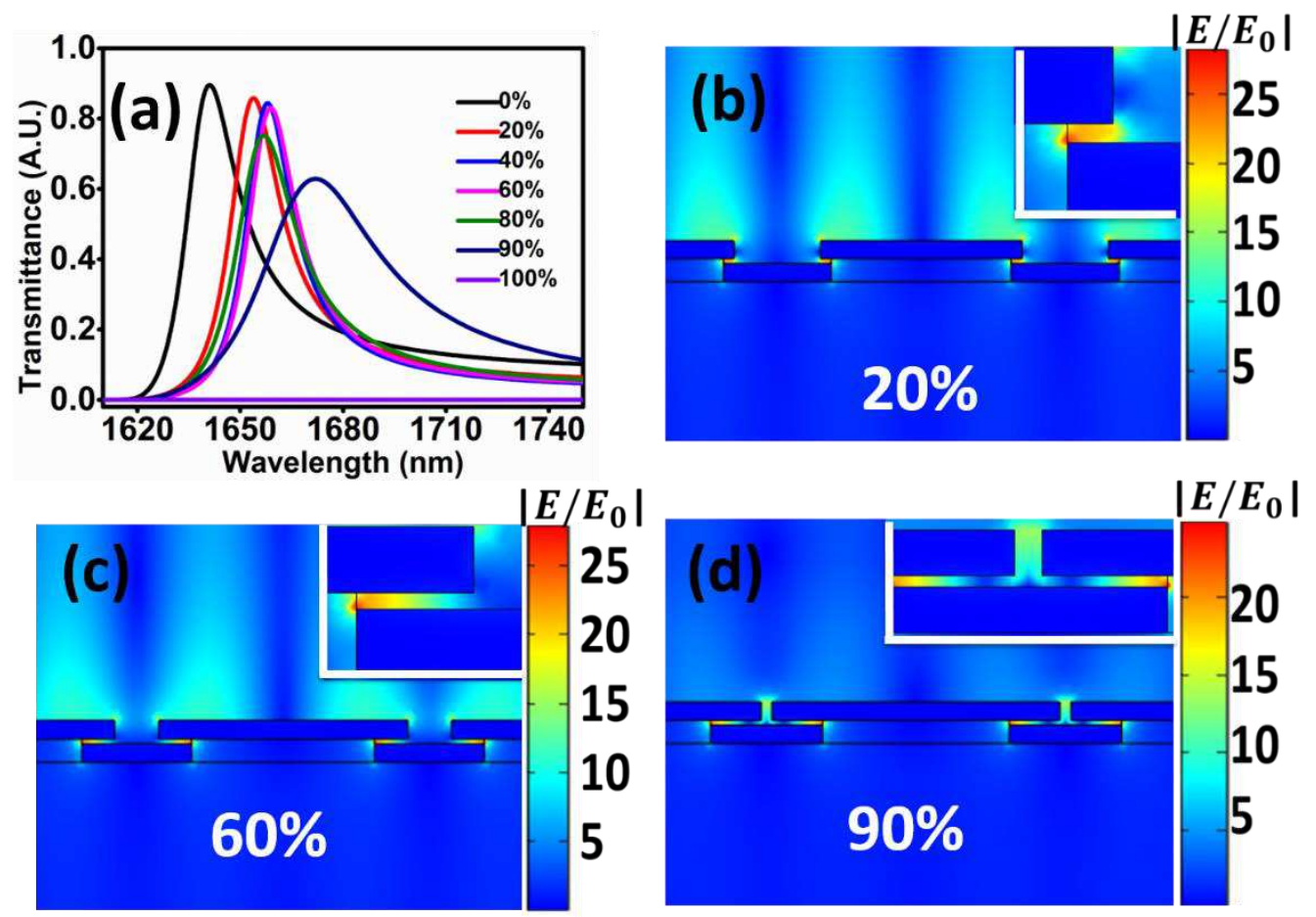

Fig. 4 a Transmission spectrum for increasing value of ' $g$ '. b-d are the electric field profiles at resonance wavelengths for 20\%,60\%, and 90\% lateral overlap, respectively. The insets of figures b-d show enlarged views of the EM field profiles confined in the hot spot between both the MNSAs.

After achieving giant extra-ordinary transmission of more than $90 \%$ through the optimal metasurface and performing control simulations on the effect of interlayer vertical distance $(\mathrm{H})$ variation on the plasmonic mode coupling between upper and lower MNSAs, we studied the impact of lateral gap of the upper slit (g) variation on the optical transmission through the metasurface by covering the lower MNSA with upper MNSA from $0 \%$ to $100 \%$ overlap measured from the edges of lower MNSA in terms of covering percentage. Till now, in the aforementioned discussions, the simulated results with $0 \%$ overlap have been presented.

Figure 4 a shows the transmittance spectra is simulated for metasurfaces having constants value of $\mathrm{h}(=100 \mathrm{~nm})$ and $\mathrm{H}(=25 \mathrm{~nm})$ and that of varying $\mathrm{g}$ in steps of $20 \%$ increase. It can be seen that transmittance maxima show a red spectral shift with an increase in the lateral overlap $(\mathrm{g})$ while the transmittance through the metasurface goes down ultimately to zero for $100 \%$ lateral overlap, when the 
opening of the slit in upper MNSA becomes zero, for obvious reasons. A clear understanding of the transmission spectra was made by studying the light confinement between upper and lower MNSAs, through the EM field profiles displayed in Fig. 4 b - d. To our surprise, about $60 \%$ transmission is achieved for about $90 \%$ closure of the slit in the upper MNSA; the credit goes to surface plasmons. However, the value of normalized electric field which is confined between upper and lower MNSAs also goes down. This study also confirms that during the fabrication of such metasurfaces, a fabrication tolerance of about $40 \%$ lateral opening does not alter the performance much, except slight variation in peak wavelength.

Fabrication tolerances in terms of the fill factor ' $f$ ' were also estimated. It is also possible to achieve good optical transmission through the proposed structure for different values of the ' $f$ ', as shown in Fig. 5 for fixed values of $\mathrm{h}, \mathrm{H}$ and $\Lambda$, which are 100, 25 and $1600 \mathrm{~nm}$, respectively.

In Fig. 5 a, we have plotted the transmittance spectra for $0^{\text {th }}$ order of the transmitted light through the plasmonic metasurface for different values of ' $f$ ', ranging from 0.625 to 0.375 in steps of 0.125 . With a decrease in the ' $f$ ', the peak resonance wavelength showed a red spectral shift. Also, the width of the resonance curve was slightly larger for $50 \%$ ' $f$ '. The aforementioned responses can be explained by understanding the roles of superstrate and substrate coupled MNSA on EM modes and their coupling, which will be evident more clearly in the subsequent discussions of Fig. 7.

We have simulated the same geometrical structure of proposed plasmonic metasurface with different noble metals $(\mathrm{Ag}, \mathrm{Al}, \mathrm{Au})$ to properly select material for the highest EOT and specified spectral response. 

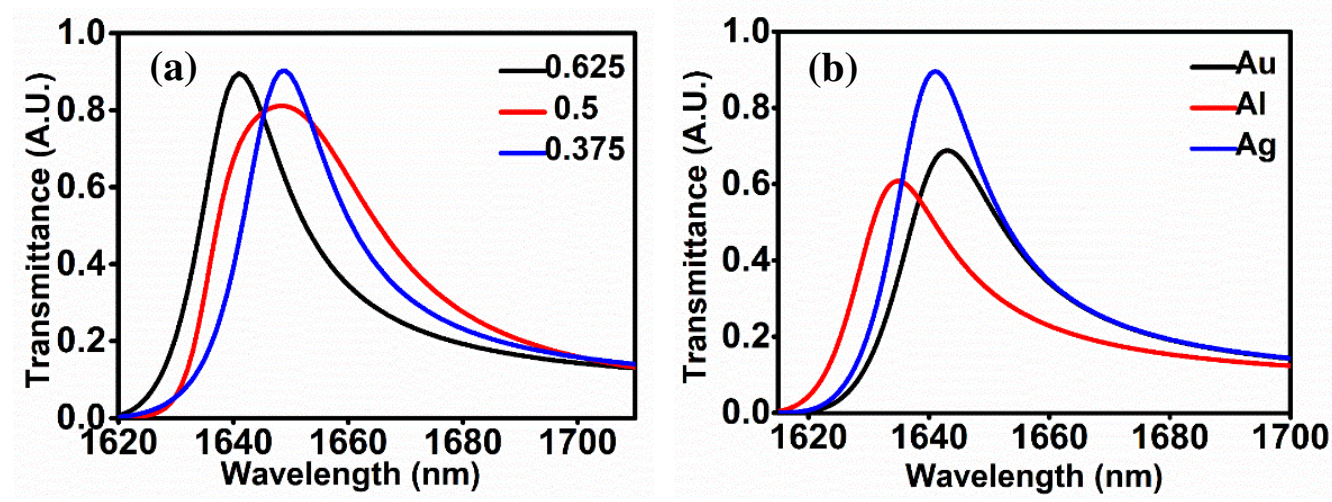

Fig. 5 Transmittance spectra for different - a values of fill factor ' $f$ '; $\mathbf{b}$ different noble metals

The values of different geometrical parameters e.g., h, $\mathrm{H}, \Lambda$, and $f$ were considered to be $100,25,1600 \mathrm{~nm}$ and 0.625 respectively for the simulation of transmission spectra with different metals.

Figure $5 \mathrm{~b}$ shows the transmission spectra for different metals used in the metasurface. It can be observed that for silver (Ag), the FWHM is the lowest while transmission peak strength is the highest as compared to that of gold $(\mathrm{Au})$ and aluminum (Al). The reason behind such a variation might be due to high values of the imaginary dielectric functions of $\mathrm{Al}$ and $\mathrm{Au}$ at the resonance wavelengths, respectively 56.61 and 13.75, as compared to that of Ag, 3.82 [69] The FWHM and the peak strength of the plasmonic response curve are the two main factors which decide the accuracy and the FOM of a plasmonic sensor. That is why Ag was considered for simulations.

Since the plasmons are excited at the metal-dielectric interface between the Ag nano- slits and the superstrate/substrate media, the plasmon resonance modes encounter a spectral shift with a change in the $\mathrm{RI}(\mathrm{s})$ of the substrate/superstrate thereby providing RI sensing ability to the metasurface. Hence, the electric and magnetic both the field profiles of the optimal metasurface were studied in detail and have been presented in Fig.6. Figures $6 a$ and $b$ respectively represent the electric and magnetic field profiles of the optimal metasurface structure having 


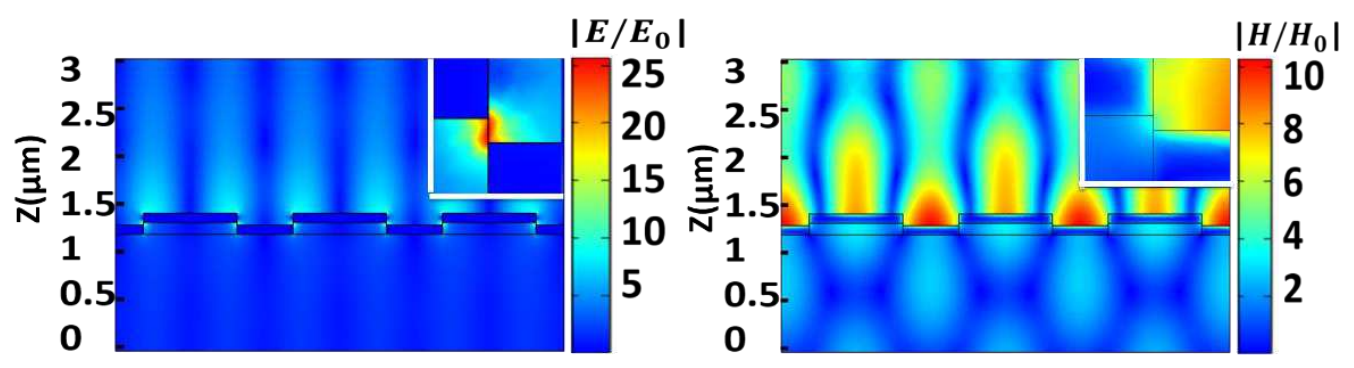

Fig.6 a Electric - b Magnetic - field profile of the optimal plasmonic metasurface at the resonance wavelength $=1641.2 \mathrm{~nm}$

geometrical parameters $\mathrm{h}, \mathrm{H}, \Lambda$, and $f$ equal to $100 \mathrm{~nm}, 25 \mathrm{~nm}, 1600 \mathrm{~nm}$ and 0.625 respectively for both the substrate and superstrate as air. The insets of both the figures show enlarged view of the field profiles in the space between both the MNSAs. It can be observed that for the optimal parameters of the metasurface at the resonance wavelength, the electric field gets confined in the space between the two MNSAs, creating a hotspot and thereby leading to giant EOT. There is relatively weak oscillating electric field in the superstrate region. However, as is evident from the Fig. 6 b, the magnetic field shows an enhanced and oscillatory behavior in the superstrate region. Though the magnitudes of the both the electric and magnetic fields are the same in the superstrate region, the penetration depth of the magnetic field is much larger as compared to electric field. Hence, the superstrate region can be used as analyte region for sensing applications.
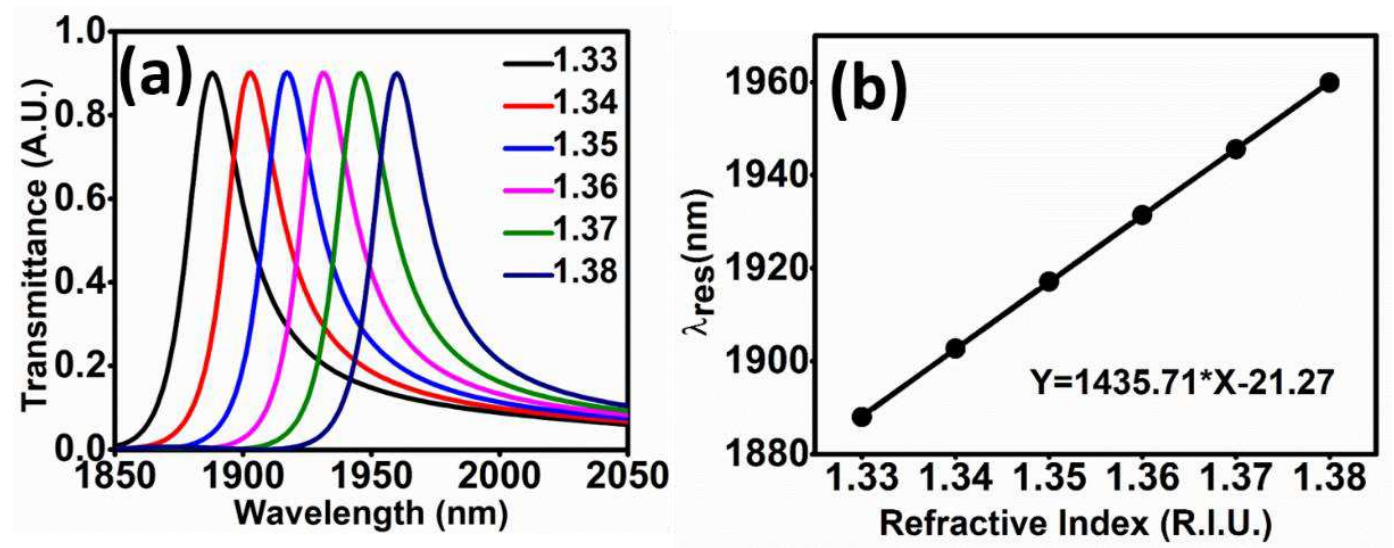

Fig.7 a Transmission spectra for varying $n_{a}$ over the metasurface based flexible sensor, b Characteristic response curve of the sensor 
For exploring the RI sensing applications of the metasurface, the superstrate was considered as analyte with refractive index $\left(n_{a}\right)$ ranging from1.33 to 1.38 in the steps of 0.01 .

PDMS was chosen as substrate material on which the metasurface was fabricated to realize an EOT-based flexible sensor. The refractive index of PDMS was taken from equation (6). The structural parameters of the metasurface were re-optimized for the maximum value of optical transmission, which was achieved to be $90.06 \%$ for the optimized values of $\mathrm{h}$ and $\mathrm{H}$ as 70 and $55 \mathrm{~nm}$, respectively, for $f=0.625$. Figure 7 a shows the transmission spectra for varying the value of $n_{a}$. It can be observed that when the value of $n_{a}$ is increased from 1.33 to 1.38 , a red shift in peak transmission spectrum is obtained. This is due to the excitation of resonance plasmonic modes at larger wavelengths for high values of $n_{a}$. In Fig. $7 \mathrm{~b}$, the values of resonance wavelengths corresponding to the highest transmittance, extracted from Fig. 7 a, have been plotted with varying $n_{a}$. A linear variation in the resonance wavelength with $n_{a}$ was observed. A sensitivity $1435.71 \mathrm{~nm} / \mathrm{RIU}$ was estimated by linear fitting of the data points and calculating the slope. The FOM was obtained to be $80 \mathrm{RIU}^{-1}$ which remained constant with the variation of $n_{a}$. This provides an added advantage over the conventional SPR, as the FWHM of the SPR curves increase with increase in analyte refractive index [75], while in the present case, the width of the plasmonic peaks remains constant.

\section{Conclusions}

In summary, we have designed, simulated, and optimized a seemingly opaque plasmonic metasurface consisting of two plasmonic MNSAs to achieve giant EOT and then used it for RI sensing applications. A design inspired for cost, labor, and time effective metasurface was first proposed and then optimized for the interlayer 
vertical distance as well as lateral overlap between both the MNSAs. The observations on the enhancement of light transmission were supported by EM field profiles. For the optimized metasurface structure, a giant EOT of more than $90 \%$ was achieved, which was attributed to the perfect coupling between the plasmonic modes generated by the upper and lower MNSAs. It was also substantiated that the plasmonic mode coupling depends on the parameters of the MNSA (e.g., thickness, fill factor etc.,) and the optimized interlayer vertical distance. An EOT based flexible RI sensor using PDMS as substrate was also studied to propose a highly sensitive metasurface based sensor. A refractive index sensitivity of 1435.71 $\mathrm{nm} / \mathrm{RIU}$ with the figure of merit (FOM) $80 \mathrm{RIU}^{-1}$ were achieved. The current study can be useful in understanding the fundamental reasons of EOT, high performance optical filters, polarizers, and flexible sensors.

\section{Author Contribution Sachin K. Srivastava (SKS) supervised this research. Both the authors} contributed to the study conception and design. Sagar K. Verma (SKV) did COMSOL simulations and developed the code. Both SKS and SKV participated in the interpretation of the data. Both the authors wrote the paper and participated in the revisions of it. Both the authors have read and approved the final version of the manuscript.

Funding This research has partially been supported by MHRD-STARS, Govt. of India (Grant No. STARS/APR2019/NS/713) and DST-INSPIRE, Govt. of India (Grant No. DST/INSPIRE/04/ 2015/002800). Sagar K. Verma acknowledges IIT Roorkee for research fellowship.

Data Availability The data that support the findings of this study are available from the corresponding author upon reasonable request.

Code Availability The code developed for this study is available from the corresponding author upon reasonable request. 


\section{Declarations}

Ethics Approval The paper reflects the authors' own original research and analysis in a truthful and complete manner. This research does NOT involve human participants or animals; thus, no ethical approval and patient consent are required.

Consent to Participate The authors provide consent to participate.

Consent for Publication The authors provide consent to publish.

Conflict of Interest The authors declare no competing interests.

\section{References}

1. Cai Y, Zhu J, Liu QH (2015) Tunable enhanced optical absorption of graphene using plasmonic perfect absorbers. Appl Phys Lett 106:1-6

2. Costantini D, Lefebvre A, Coutrot AL, Moldovan-Doyen I, Hugonin JP, Boutami S, Marquier F, Benisty H, Greffet JJ (2015) Plasmonic metasurface for directional and frequency-selective thermal emission. Phys Rev Appl 4:1-6

3. López-Muñoz GA, Estevez MC, Peláez-Gutierrez EC, Homs-Corbera A, García-Hernandez MC, Imbaud JI, Lechuga LM (2017) A label-free nanostructured plasmonic biosensor based on Blu-ray discs with integrated microfluidics for sensitive biodetection. Biosens Bioelectron 96:260-267

4. Qin F, Ding L, Zhang L, Monticone F, Chum CC, Deng J, Mei S, Li Y, Teng J, Hong M, Zhang S, Alù A, Qiu CW (2016) Hybrid bilayer plasmonic metasurface efficiently manipulates visible light. Sci Adv 2:1-9

5. Reshef O, Huttunen MJ, Carlow G, Sullivan BT, Dolgaleva K, Boyd RW (2019) Multiresonant high - Q plasmonic metasurfaces. Nano Lett 19:6429-6434

6. Wang S, Zhan Q (2016) Reflection type metasurface designed for high efficiency vectorial field generation. Sci Rep 6:1-8 
7. Sun S, He Q, Xiao S, Xu Q, Li X, Zhou L (2012) Gradient-index metasurfaces as a bridge linking propagating waves and surface waves. Nat Mater 11:426-431

8. Lin J, Mueller JPB, Wang Q, Yuan G, Antoniou N, Yuan XC, Capasso F (2013) Polarization-controlled tunable directional coupling of surface plasmon polaritons. Science 340:331-334

9. Rodríguez-fortuño FJ, Martínez A, Wurtz G a, Zayats A V (2013) Electromagnetic guided modes. Science 340:328-331

10. Huang L, Chen X, Bai B, Tan Q, Jin G, Zentgraf T, Zhang S (2013) Helicity dependent directional surface plasmon polariton excitation using a metasurface with interfacial phase discontinuity. Light Sci Appl 2:1-7

11. Shitrit N, Yulevich I, Maguid E, Ozeri D, Veksler D, Kleiner V, Hasman E (2013) Spin-optical metamaterial route to spin-controlled photonics. Science 340:724-726

12. Pendry JB, Schurig D, Smith DR (2006) Controlling electromagnetic fields. Science 312:1780-1782

13. Liu Y, Zentgraf T, Bartal G, Zhang X (2010) Transformational plasmon optics. Nano Lett 10:1991-1997

14. Huidobro PA, Nesterov ML, Martín-Moreno L, García-Vidal FJ (2010) Transformation optics for plasmonics. Nano Lett 10:1985-1990

15. Aubry A, Lei DY, Fernández-Domínguez AI, Sonnefraud Y, Maier SA, Pendry JB (2010) Plasmonic light-harvesting devices over the whole visible spectrum. Nano Lett 10:2574-2579

16. Zhu J, Wang Z, Lin S, Jiang S, Liu X, Guo S (2020) Low-cost flexible plasmonic nanobump metasurfaces for label-free sensing of serum tumor marker. Biosens Bioelectron 150:1-7 
17. Bin-alam MS, Reshef O, Mamchur Y, Alam MZ, Carlow G, Upham J, Sullivan BT, Ménard J, Huttunen MJ, Boyd RW, Dolgaleva K (2021) Ultra-high-Q resonances in plasmonic metasurfaces. Nat Commun 12:1-8

18. Nie S, Emory SR (1997) Probing single molecules and single nanoparticles by surface-enhanced Raman scattering. Science 275:1102-1106

19. Willets KA, Van Duyne RP (2007) Localized surface plasmon resonance spectroscopy and sensing. Annu Rev Phys Chem 58:267-297

20. Azzam SI, Kildishev A V., Ma RM, Ning CZ, Oulton R, Shalaev VM, Stockman MI, Xu JL, Zhang X (2020) Ten years of spasers and plasmonic nanolasers. Light Sci Appl 9:1-21

21. Ren M, Jia B, Ou JY, Plum E, Zhang J, MacDonald KF, Nikolaenko AE, Xu J, Gu M, Zheludev NI (2011) Nanostructured plasmonic medium for terahertz bandwidth all-optical switching. Adv Mater 23:5540-5544

22. Kauranen M, Zayats A V. (2012) Nonlinear plasmonics. Nat Photonics $6: 737-748$

23. Yu N, Genevet P, Kats M a, Aieta F, Tetienne J-P, Capasso F, Gaburro Z (2011) Light propagation with phase reflection and refraction. Science $334: 333-337$

24. Won R (2017) View from... SPP8: The rise of plasmonic metasurfaces. Nat Photonics 11:462-464

25. Meinzer N, Barnes WL, Hooper IR (2014) Plasmonic meta-atoms and metasurfaces. Nat Photonics 8:889-898

26. Ebbesen TW, Kuipers L (2010) Light passing through subwavelength apertures. 82:729-787

27. Sun S, Leggett GJ (2004) Matching the resolution of electron beam lithography by scanning near-field photolithography. Nano Lett 4:1381- 
28. Neumann L, Pang Y, Houyou A, Juan ML, Gordon R, Van Hulst NF (2011) Extraordinary optical transmission brightens near-field fiber probe. Nano Lett 11:355-360

29. You A, Be MAY, In I (1998) density data storage Near-field. 61:141-144

30. Shinada S, Hashizume J, Koyama F (2003) Surface plasmon resonance on microaperture vertical-cavity surface-emitting laser with metal grating. Appl Phys Lett 83:836-838

31. Hashizume J, Koyama F (2004) Plasmon-enhancement of optical near-field of metal nanoaperture surface-emitting laser. Appl Phys Lett 84:3226-3228

32. Ohno T, Bain JA, Schlesinger TE (2007) Observation of geometrical resonance in optical throughput of very small aperture lasers associated with surface plasmons. J Appl Phys 101:1-5

33. Onishi T, Tanigawa T, Ueda T, Ueda D (2007) Polarization control of vertical-cavity surface-emitting lasers by utilizing surface plasmon resonance. IEEE J Quantum Electron 43:1123-1128

34. Nylander C, Liedberg B, Lind T (1982) Gas detection by means of surface plasmon resonance. Sensors and Actuators 3:79-88

35. Tetz KA, Pang L, Fainman Y (2006) High-resolution surface plasmon resonance sensor based on linewidth-optimized nanohole array transmittance. Opt Lett 31:1528

36. Lesuffleur A, Kumar LKS, Brolo AG, Kavanagh KL, Gordon R (2007) Apex-enhanced Raman spectroscopy using double-hole arrays in a gold film. J Phys Chem C 111:2347-2350

37. Reilly TH, Chang S, Corbman JD, Schatz GC, Rowlen KL (2007) Quantitative evaluation of plasmon enhanced raman scattering from 
nanoaperture arrays. J Phys Chem C 111:1689-1694

38. Williams SM, Stafford AD, Rodriguez KR, Rogers TM, Coe J V. (2003) Accessing surface plasmons with Ni microarrays for enhanced ir absorption by monolayers. J Phys Chem B 107:11871-11879

39. Dintinger J, Klein S, Ebbesen TW (2006) Molecule-surface plasmon interactions in hole arrays: Enhanced absorption, refractive index changes, and all-optical switching. Adv Mater 18:1267-1270

40. Liu Y, Blair S (2003) Fluorescence enhancement from an array of subwavelength metal apertures. Opt Lett 28:507-509

41. Liu Y, Blair S (2004) Fluorescence transmission through 1-D and 2-D periodic metal films. Opt Express 12:3686-3693

42. Rigneault H, Capoulade J, Dintinger J, Wenger J, Bonod N, Popov E, Ebbesen TW, Lenne PF (2005) Enhancement of single-molecule fluorescence detection in subwavelength apertures. Phys Rev Lett 95:1-4

43. Wenger J, Gérard D, Dintinger J, Mahboub O, Bonod N, Popov E, Ebbesen TW, Rigneault H (2008) Emission and excitation contributions to enhanced single molecule fluorescence by gold nanometric apertures. Opt Express $16: 3008-3020$

44. Drezet A, Genet C, Laluet J-Y, Ebbesen TW (2008) Optical chirality without optical activity: How surface plasmons give a twist to light. Opt Express 16:12559-12570

45. Drezet A, Genet C, Ebbesen TW (2008) Miniature plasmonic wave plates. Phys Rev Lett 101:1-4

46. Anishur Rahman ATM, Majewski P, Vasilev K (2012) Extraordinary optical transmission: coupling of the Wood-Rayleigh anomaly and the Fabry-Perot resonance. Opt Lett 37:1742-1744 
47. Li W-D, Hu J, Chou SY (2011) Extraordinary light transmission through opaque thin metal film with subwavelength holes blocked by metal disks. Opt Express 19:21098

48. Bethe H.A. (1944) Theory of diffraction by small holes. Phys Rev 66:163182

49. Roberts A (1987) Electromagnetic theory of diffraction by a circular aperture in a thick, perfectly conducting screen. 4:1970-1983

50. Huang C, Kodis RD, Levine H (1955) Diffraction by apertures. J Appl Phys 26:151-165

51. Mendez OM, Cadilhac M, Petit R (1983) Diffraction of a Two-dimensional electromagnetic beam wave by a thick slit pierced in a perfectly conducting screen. J Opt Soc Am 73:328-331

52. Porto JA, Garc1 FJ (1999) Transmission resonances on metallic gratings with very narrow slits. 83:2845-2848

53. Liu H, Lalanne P (2008) Microscopic theory of the extraordinary optical transmission. Nature 452:728-731

54. Lalanne P, Hugonin JP, Rodier JC (2005) Theory of surface plasmon generation at nanoslit apertures. Phys Rev Lett 95:1-4

55. Lalanne P, Hugonin JP, Liu HT, Wang B (2009) A microscopic view of the electromagnetic properties of sub- $\lambda$ metallic surfaces. Surf Sci Rep 64:453469

56. Dahlin A, Zäch M, Rindzevicius T, Käll M, Sutherland DS, Höök F (2005) Localised surface plasmon resonance sensing of lipid-membrane-mediated biorecognition events. J Am Chem Soc 127:5043-5048

57. Rindzevicius T, Alaverdyan Y, Dahlin A, Höök F, Sutherland DS, Käll M (2005) Plasmonic sensing characteristics of single nanometric holes. Nano 
Lett 5:2335-2339

58. Srivastava SK, Verma R, Gupta BD (2016) Theoretical modeling of a selfreferenced dual mode SPR sensor utilizing indium tin oxide film. Opt Commun 369:131-137

59. Karabchevsky A (2011) Dual-surface plasmon excitation with thin metallic nanoslits. J Nanophotonics 5:1-9

60. Karabchevsky A, Krasnykov O, Auslender M, Hadad B, Goldner A, Abdulhalim I (2009) Theoretical and experimental investigation of enhanced transmission through periodic metal nanoslits for sensing in water environment. Plasmonics 4:281-292

61. Srivastava SK, Abdulhalim I (2015) Self-referenced sensor utilizing extraordinary optical transmission from metal nanoslits array. Opt Lett 40:24252428

62. Shen Y, Liu M, Li J, Chen X, Xu HX, Zhu Q, Wang X, Jin C (2012) Extraordinary transmission of three-dimensional crescent-like holes arrays. Plasmonics 7:221-227

63. Koerkamp KJK, Enoch S, Segerink FB, Van Hulst NF, Kuipers L (2004) Strong influence of hole shape on extraordinary transmission through periodic arrays of subwavelength holes. Phys Rev Lett 92:18-21

64. Kitson SC, Barnes WL, Sambles JR (1996) Full photonic band gap for surface modes in the visible. Phys Rev Lett 77:2670-2673

65. Barnes WL, Dereux A, Ebbesen TW (2003) Surface plasmon subwavelength optics. Nature 424:824-830

66. Abutoama M, Bajaj A, Li D, Wang Y, Jiang L, Abdulhalim I (2020) Resonant modes of reflecting gratings engineered for multimodal sensing. APL Photonics 5:1-13 
67. Cao J, Sun Y, Kong Y, Qian W (2019) The sensitivity of grating-based SPR sensors with wavelength interrogation. Sensors (Switzerland) 19:1-9

68. Wang X, Zhu J, Xu Y, Qi Y, Zhang L, Yang H, Yi Z (2021) A novel plasmonic refractive index sensor based on gold/silicon complementary grating structure. Chinese Phys B 30:024207

69. P. B. Johnson and R. W. Christy (1972) Optical constants of the nobel metals. Phys Rev B 6:4370-4379

70. Zeng L, Chen M, Yan W, Li Z, Yang F (2020) Si-grating-assisted SPR sensor with high figure of merit based on Fabry-Pérot cavity. Opt Commun $457: 1-7$

71. Chen D, Chen F, Hu X, Zhang H, Yin X, Zhou Y (2015) Thermal stability, mechanical and optical properties of novel addition cured PDMS composites with nano-silica sol and MQ silicone resin. Compos Sci Technol 117:307-314

72. Cai DK, Neyer A, Kuckuk R, Heise HM (2008) Optical absorption in transparent PDMS materials applied for multimode waveguides fabrication. Opt Mater (Amst) 30:1157-1161

73. Schneider F, Draheim J, Kamberger R, Wallrabe U (2009) Process and material properties of polydimethylsiloxane (PDMS) for optical MEMS. Sensors Actuators, A Phys 151:95-99

74. Abutoama M, Abdulhalim I (2015) Self-referenced biosensor based on thin dielectric grating combined with thin metal film. Opt Express 23:2866728682

75. Srivastava SK, Abdulhalim I (2015) Spectral Interrogation based SPR Sensor for Blood Glucose Detection with Improved Sensitivity and Stability. J Biosens Bioelectron 06:10-12 
